\title{
Protocol Sequence Based Wireless Media Access Control in Networked Control Systems
}

\author{
Hui Cheng* \\ Dept. of Automation \\ Sun Yat-sen University \\ Guangzhou, China
}

\author{
Yi Chen*, Wing Shing Wong* \\ Dept. Information Engineering \\ The Chinese University of Hong Kong \\ Hong Kong, China
}

\author{
Qiong Yang", Lianfeng Shen \\ National Mobile Comm. Research Lab \\ Southeast University \\ Nanjing, China
}

\begin{abstract}
In some real-time networked control applications, information between sensors and controllers is exchanged over a shared wireless channel. One key issue is to manage multiple access to the shared medium to accomplish different control tasks. In the paper, a protocol sequence based media access control (MAC) design is presented for networked control systems (NCSs). It is challenging to design an optimal or suboptimal controller since the sensor packets and control packets could be lost in unreliable wireless networks. An ad hoc and efficient control policy is presented. Numerical results illustrate that the cost performance of the protocol sequence based NCS is much better than that of the $\pi$-persistent random access based NCS.
\end{abstract}

Keywords-networked control systems, media access control, ptotocol sequence, lossy networks, linear qudratic control

\section{INTRODUCTION}

In time-critical network control applications, sensor data and control values, which we call time-critical data traffic, are transmitted over unreliable wireless networks. The timecritical data traffic can only tolerate low bit error probability and constrained delays [1-2]. Due to data packet drops and communication delay inherent in networked control systems (NCSs), numerous works have been devoted to develop quantization and coding schemes, control laws as well as communication protocols for NCSs [3-4]. In contrast to pointto-point topology, controlling multiple plants over the shared wireless network can provide significant improvement in efficiency and reduction of development cost. It is thus of interests to study NCSs with information between multiple dynamical plants and controllers being exchanged over the shared wireless network.

Due to the broadcast nature of wireless channels, a major challenge is to schedule network resources of the shared wireless network to accomplish various control tasks. This is the function of the Media Access Control (MAC) protocol layer. A suitable MAC protocol is required to moderate the access of simultaneous systems in an efficient manner. In the literature, the MAC protocols can be generally categorized

\footnotetext{
*This research is supported by project MMT-p3-11 of the Shun Hing Institute of Advanced Engineering, CUHK, and National Natural Science Foundation of China under Grants 60804012 and 61174060.

*The authors are also with the Shun Hing Institute of Advanced Engineering and Dept. of Information Engineering, CUHK.
}

into two categories, centralized and distributed [7], each of which has its own challenges. A typical centralized protocol is time division multiple access (TDMA). TDMA can guarantee transmission, while it needs centralized coordination and slot synchronization. Traditional distributed protocols include ALOHA and carrier sense multiple access (CSMA) [7]. In ALOHA, packets that collide will be retransmitted at a random time in the future. In CSMA, a user listens to the channel before transmitting, and if the channel is busy, it delays access and retries later. Although ALOHA and CSMA do not need centralized coordination, they require complex processing such as back off algorithm and random number generation, and need frequent monitoring of the channel. Traditional MAC mechanisms mentioned above were considered for NCSs, and the system performance was studied under these classical MAC protocols [5-7].

While some of the traditional MAC protocols including ALOHA and CSMA can provide excellent system throughput performance, they cannot guarantee finite delay bounds. In time-critical applications, one tantalizing approach is provided by protocol sequences. New families of protocol sequences were discovered [8-11], which can be used to provide practical solutions for the MAC protocol of wireless networks. To guarantee that the maximum latency is bounded within one sampling interval, the idea of protocol sequences will be deployed for the MAC design. The scheduling of packets is achieved by assigning each dynamical system a protocol sequence. Each plant reads out the bits from the assigned protocol sequence periodically, and sends the packet if and only if the value is equal to one. According to different performance guarantee criteria, there are different construction methods for protocol sequence. In the paper, we focus on Generalized Prime Sequences (GPS) [11]. The performance of a NCS is investigated under two types of MAC protocols: GPS and random access based strategies.

The paper is organized as follows. Section II describes the problem setting. Section III presents protocol sequence based MAC design. State estimation and control law are presented in Section IV. Numerical results are presented in Section V. Section VI concludes the paper.

\section{PROBLEM ForMULATION}

Consider a NCS, where the information change between multiple distributed plants and controllers is transmitted over 


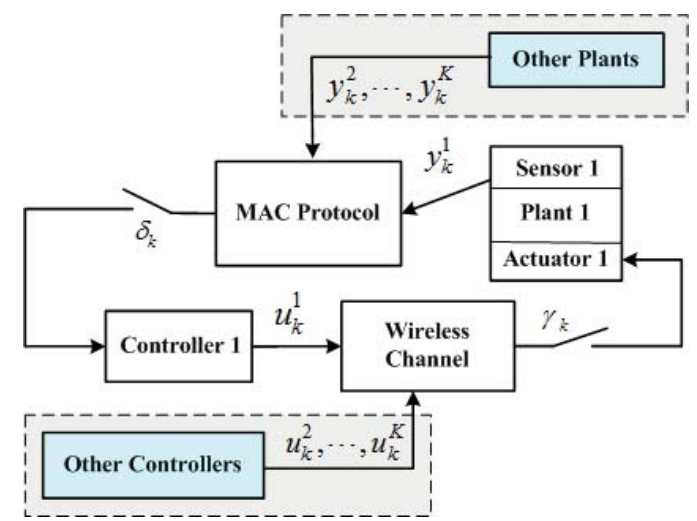

Fig. 1. Diagram of a networked control system with MAC protocols.

the shared wireless network. As multiple controllers and plants share the common wireless network, suitable MAC protocols are required to arbitrate access to the shared medium. Fig. 1 illustrates the diagram of a NCS with MAC mechanisms managing the medium access contention among multiple plants and controllers. There are $K$ pairs of plants-controllers. The MAC protocol is performed in the sensor node. The switches "open" or "closed" status indicates the medium access status. Due to the contention among multiple plants and controllers over the shared network as well as the thermal noise, both the sensor and the control packets may be dropped in transmitting over the unreliable wireless network. Fig. 2 depicts the diagram of a control loop closed through the lossy wireless network.

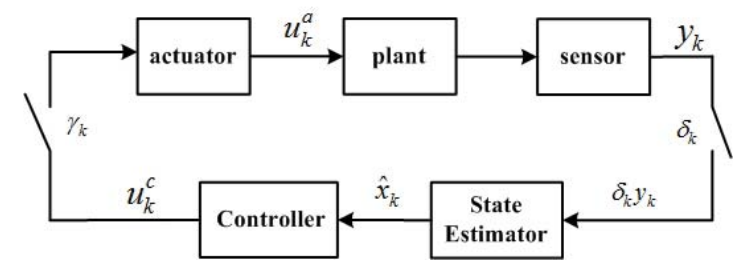

Fig. 2. Diagram of a control system closed through a lossy network.

For simplicity, assume that the multiple plants have the same dynamics. For each plant, consider the following linear stochastic system with intermittent sensor and control packets:

$$
\begin{gathered}
\boldsymbol{x}_{k+1}=\boldsymbol{A} \boldsymbol{x}_{k}+\boldsymbol{B} \boldsymbol{u}_{k}^{a}+\boldsymbol{w}_{k}, \\
\boldsymbol{u}_{k}^{a}=\gamma_{k} \boldsymbol{u}_{k}^{c}+\left(1-\gamma_{k}\right) \boldsymbol{u}_{k-1}^{a}, \\
\boldsymbol{y}_{k}=\delta_{k} \boldsymbol{C} \boldsymbol{x}_{k}+\boldsymbol{v}_{k},
\end{gathered}
$$

where $\boldsymbol{A}$ is the state transition matrix, $\boldsymbol{B}$ is the input matrix, $\boldsymbol{C}$ is the output matrix, $\boldsymbol{u}_{k}^{c}$ is the computed control value, $\boldsymbol{u}_{k}^{a}$ is the control input to the actuator, $\left(\boldsymbol{x}_{0}, \boldsymbol{w}_{k}, \boldsymbol{v}_{k}\right)$ denote uncorrelated white Gaussian vectors with mean $\left(\overline{\boldsymbol{x}}_{0}, \boldsymbol{0}, \boldsymbol{0}\right)$ and covariance $\left(\boldsymbol{P}_{0}, \boldsymbol{Q}, \boldsymbol{R}\right)$, and $\left(\delta_{k}, \gamma_{k}\right)$ represent i.i.d. Bernoulli random variables with $P\left(\delta_{k}=1\right)=\bar{\delta}$ and $P\left(\gamma_{k}=1\right)=\bar{\gamma}$. The stochastic variable $\delta_{k}$ models the packet loss between the sensor and the controller; while the variable $\gamma_{k}$ models the packet loss between the controller and the actuator.

As shown in (2), the hold-input control strategy is adopted.
If the control packet at the $k$ th time is successfully delivered, the control input to the actuator $u_{k}^{a}$ is the computed control value $u_{k}^{c}$. On the other hand, when the control packet is lost, the last control input is applied to the actuator, i.e., $u_{k}^{a}=u_{k-1}^{a}$.

Consider the infinite horizon problem that minimizes the average stage cost of a linear stochastic system over lossy networks. The objective quadratic function is given by:

$$
J=\lim _{M \rightarrow \infty} \frac{1}{M} E\left[\sum_{m=0}^{M-1}\left(\boldsymbol{x}_{m}^{T} \boldsymbol{Q}_{1} \boldsymbol{x}_{m}+\boldsymbol{u}_{m}^{a T} \boldsymbol{Q}_{2} \boldsymbol{u}_{m}^{a}\right)\right],
$$

where $\boldsymbol{Q}_{1}$ and $\boldsymbol{Q}_{2}$ are positive definite weighting matrices. We aim to find $\boldsymbol{u}_{0}^{a}, \boldsymbol{u}_{1}^{a}, \ldots$ to minimize the cost $J$.

\section{Wireless Media Access Control Protocols}

As multiple distributed plants and controllers share the common wireless network, suitable MAC protocols are required to arbitrate access to the shared medium. We will apply the idea of protocol sequence for the time-critical network control applications. The MAC protocol is performed in the sensor node. The packets scheduling is done by assigning each plant a protocol sequence. Each plant reads out the bits from the assigned protocol sequence periodically, and transmits the packet if and only if the bit value is equal to one. The performance in terms of probability of failure delivery of the protocol sequence based MAC scheme will be studied and compared with that of the $\pi$-persistent random access scheme.

For the convenience of description, we use plant to refer to the union of the actuators, plant and sensors, and use controller to refer to the union of the state estimator and controller. The communications from the plants to the controllers are called uplink, while the communications from the controllers to the plants are called downlink. Assume that there is a pair of orthogonal channels for uplink and downlink communications, respectively. All the plant-controller pairs share the same uplink channel and the same downlink channel. The channel is divided into small time slots of equal duration, and a packet transmission is done within a time slot. At any time slot, if more than one plant transmits, a collision occurs and all transmitted packets in that time slot are lost.

For each feedback control system closed through the network, the target is that within one sampling period, the plant successfully delivers a sensor data packet to the remote controller over the uplink channel, and receives a control packet from the controller over the downlink channel. It needs to be mentioned that the downlink communication is eventdriven, that is, the controller starts to transmit only when it receives a packet from the plant. Therefore, we only need to consider the MAC for the uplink transmission. Moreover, it is noted that the control packet received by the plant in fact can be served as acknowledgment (ACK). Hence, in the following discussed MAC protocols, we set the rule that once a plant receives the control packet (referred to as ACK) it will keep silent in the remaining time slots of that sampling period. If a plant does not receive the control packet within a sampling period, a failure delivery is counted. 


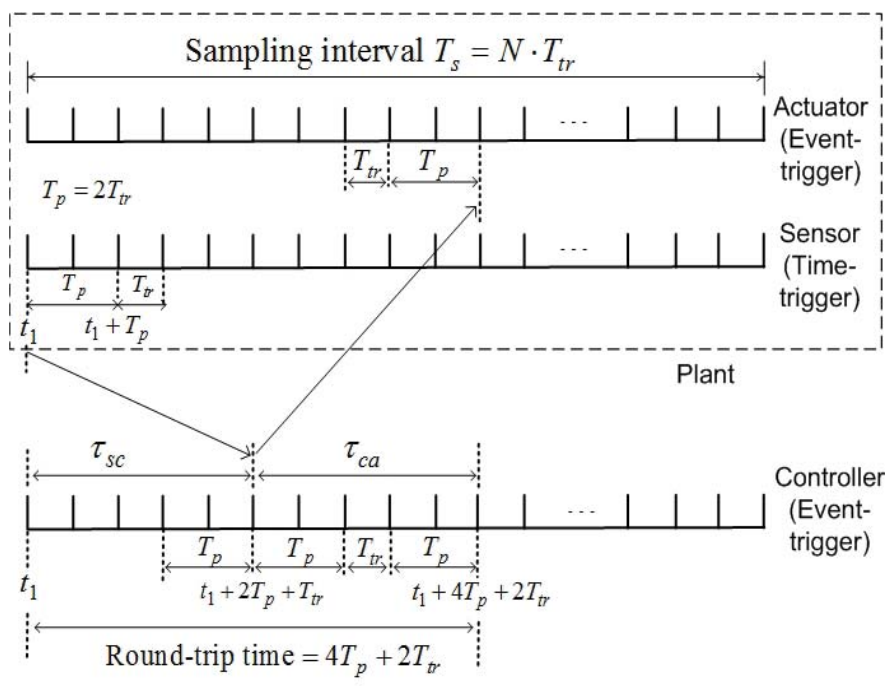

Fig. 3. Time diagram of a NCS in a sampling interval.

The time slots of all users are synchronized while the boundaries of the sampling periods are not necessarily aligned to each other. Fig. 3 illustrates the time diagram of a NCS. $N$ denotes the number of time slots in a sampling period. Assume that the slot time is equal to the transmission time $T_{t r}$. Let $T_{p}$ denote the processing time, cost by the micro control unit (MCU) to manipulate the sample data and transmit them to the antenna. The processing time depends on the device characteristics and can not be ignored in practical applications [12]. In many real time applications, the sensor data packets are short, and the transmission time is generally less than the processing time. Assume $T_{p}=m T_{t r}$ for some integers $m$. An example of the time diagram for $T_{p}=2 T_{t r}$ is shown in Fig. 3 .

Let $t_{1}$ denote the start of a sampling period. First at the sensor side, the sensor data arrives at the antenna of the sensor at $t_{1}+T_{p}$. After $T_{t r}$ time, the sensor data arrives at the antenna of the controller. Again at the controller side, it takes $T_{p}$ for the MCU at the controller to receive the sensor data from the antenna. Assume that the computational time of the CPU can be neglected compared with the transmission time. Therefore, the control data is ready at the MCU once the sensor data is received, that is, at $t_{1}+2 T_{p}+T_{t r}$. The transmission procedure from the controller to the actuator follows the same rule and is not repeated here. A round-trip time from the sensor to the actuator is hence $4 T_{p}+2 T_{t r}$ for each plant. Define $T_{a c k}$ be the time for the antenna of the plant to detect the control packet (served as ACK) after transmitting the sensor data without packet drops (ref. Fig. 4). It follows as

$$
T_{a c k}=3 T_{p}+T_{t r} .
$$

Note that if a sensor packet is transmitted within the last $T_{a c k}$ of a sampling period, its ACK would be received beyond the same sampling period and become useless. Hence, the feasible time for transmitting sensor packets in a sampling period should exclude the front $T_{p}$ and the last $T_{a c k}$, as shown in Fig. 4. The total number of feasible slots is $N_{\text {fes }}=N-$ $\left(T_{a c k}+T_{p}\right) / T_{t r}$. Furthermore, due to the processing time, the actual transmission slots should be separated by $m$ slots. The total feasible transmission slots is hence $N_{t r}=\left\lceil N_{\text {fes }} / \mathrm{m}\right\rceil$.

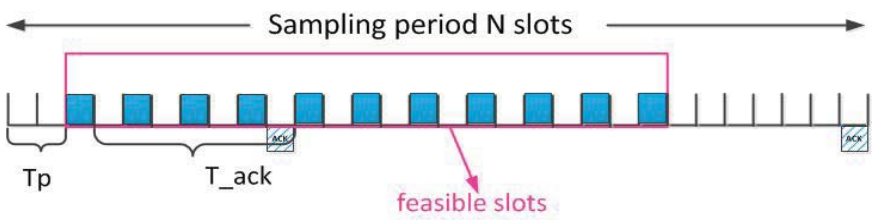

Fig. 4. Time slots arrangement for the MAC in a sampling period.

Let $P_{\text {noise }}$ be the probability of packet loss due to thermal noise (collision free cases). $P_{u p}$ represents the probability of fail uplink transmission, that is, a controller does not receive any packet from its intended plant in a sampling period. $P_{\text {round }}$ is the probability of fail round-trip communication, that is, a plant does not receive any packet from its intended controller in a sampling period. In the following, the generalized protocol sequences (GPS) based MAC with ACK is introduced.

\section{A. Generalized Protocol Sequences (GPS) with ACK}

The construction of GPS uses the modulo- $p$ multiplication for a prime number $p$ [11]. Let $\operatorname{rem}(z, p)$ denote the remainder when integer $z$ is divided by $p$. For a given prime number $p$ and an integer $q$ with $q \geq p$, the protocol sequences with period $L=p q$ and hamming weight $p$ (number of ones in the sequence) are constructed as follows:

$$
I_{g}=\{\operatorname{rem}(g l, p)+l q: l=0,1,2, \ldots, p-1\},
$$

where $g=0,1, \ldots, p-1$, indicate the $g$ th sequence out of the total $p$ sequences. $I_{g}$ specifies the location of " 1 " in the $g$ th sequence. Take $p=3$ and $q=3$ for example, it obtains $I_{0}=\{0,3,6\}, I_{1}=\{0,4,8\}$ and $I_{2}=\{0,5,7\}$. The corresponding protocol sequences are given as:

$$
\begin{array}{lllllllllll}
\text { PS1 : } 1 & 0 & 0 & 1 & 0 & 0 & 1 & 0 & 0 & 0 \\
\text { PS2 : } & 0 & 0 & 0 & 0 & 1 & 0 & 0 & 0 & 1, \\
\text { PS3 : } & 1 & 0 & 0 & 0 & 0 & 1 & 0 & 1 & 0 .
\end{array}
$$

It is noted that the first sequence is superior to other sequences as " 1 "s are evenly distributed. We will observe this phenomenon later in the simulation results.

As we have mentioned earlier, due to the processing time, the feasible transmission slots should be separated by $m$ slots. Therefore, the actual used sequences for MAC access are modified version of the protocol sequences by inserting $m-1$ zeros after each bit. Take $m=2$ for example, the access sequence corresponding to PS1 is

AS1: $10 \underline{00} \underline{00} \underline{10} \underline{00} \underline{00} \underline{10} \underline{00} \underline{00 .}$

Each plant is assigned a distinct access sequence and accesses the channel according to the sequence pattern: one 
for delivery and zero for silence. Once the plant receives the ACK, it remains silent from next time slot till the end of the sampling period, irrespective of the ones in the rest part of the sequence. It needs to be mentioned that the period $L$ of GPS cannot be chosen arbitrary, since $p$ must be a prime number and $q \geq p$. Given $K$ plants, $p$ is chosen as the prime number satisfying $p \geq K$. Let $q=p$ so $L=p^{2}$. When $L \geq N_{t r}$, only the first $N_{t r}$ bits in the GPS are used. For example, in the case of $N_{t r}=6$, the sequences used for MAC can be reduce to be

$$
\begin{aligned}
& \text { AS1: } 100000100000 \text {, } \\
& \text { AS2: } 100000001000 \text {, } \\
& \text { AS3 : } 100000000010 \text {. }
\end{aligned}
$$

When $L<N_{t r}$, repeat the sequences until $N_{t r}$ slots are filled.

Fig. 5 illustrates the procedure of packet transmission using GPS with $N=14, T_{p}=T_{t r}, T_{a c k}=4 T_{t r}$ and $L=N_{\text {fes }}$ $=9$. The first plant transmits a packet at the $2^{\text {nd }}$ slot. At the $5^{\text {th }}$ slot, since the ACK has not been received yet, the first plant transmits again. At the $6^{\text {th }}$ slot, the first plant receives the ACK and will not transmit at the $8^{\text {th }}$ slot. The third plant collides with the first plant at the $5^{\text {th }}$ slot. Therefore, the third plant does not receive the ACK at the $9^{\text {th }}$ slot and retransmits at the $10^{\text {th }}$ and $12^{\text {th }}$ slot. Note that the packet loss not only results from collision but also results from thermal noise. For example, at the $8^{\text {th }}$ slot, only the second plant transmits and there is no collision. But due to the thermal noise, the packet drops at either the uplink (with probability $P_{n o i s e}$ ) or downlink

(with probability $P_{\text {noise }}$ ) channel so that it does not receive the ACK at the $12^{\text {th }}$ slot.

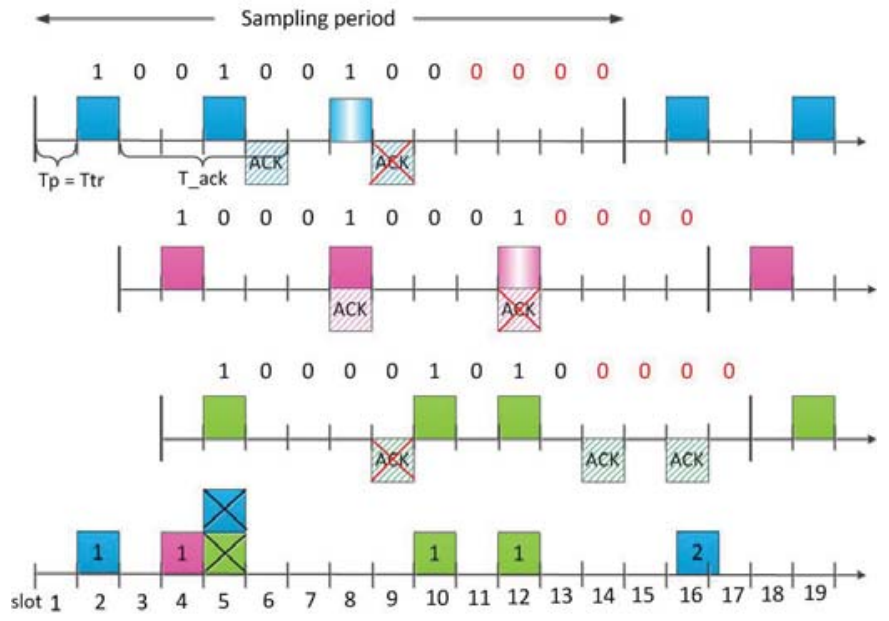

Fig. 5. The procedure of packet transmission using GPS.

\section{B. Random Access (RA) with ACK}

A simple form of random access (RA) with ACK, called $\pi$-persistent random access, is considered. In each feasible transmission time slot $\left(N_{t r}\right)$, each plant accesses the channel independently with probability $\pi$. Once a plant receives the $\mathrm{ACK}$, it remains silent from next time slot till the end of the sampling period. For fair comparison with the GPS, we set $\pi=p / L$ so that the average number of access times for the RA scheme is the same as that of the GPS scheme (the number of ones in a sequence in a GPS).

\section{STATE Estimation AND LQG CONTROL}

The linear quadratic Gaussian (LQG) control problem in the case where both observation and control packets may be dropped over wireless networks were analyzed in [13], where Transmission Control (TCP)-like and User Datagram (UDP)like protocols were considered. In TCP-like case, successful or unsuccessful packets delivery at the receiver is acknowledged to the sender within the same sampling period. While in UDPlike protocols, no such feedback is provided. It is shown in [13] that the classic separation principle holds for the TCP-like protocols. The estimator and the controller hence can be designed independently. On the contrast, the classic separation principle does not hold for the UDP-like case, and the LQG optimal controller is in general nonlinear. A suboptimal linear controller for UDP-like networked system is presented in [14], where the iterative solution of six coupled nonlinear equations converges to the fixed point solution under some hypotheses. In practice, the acknowledgement packets may be lost in TCPlike case. In [15], it is shown that even partial lack of acknowledgement of control packets results in the failure of the separation principle. Since estimation and control are coupling for UDP case and TCP-like protocols with partial acknowledgement, design of the optimal/suboptimal estimator and controller presents a highly complex problem [14][15] and remains an interesting issue to be developed.

It should be noted hat the acknowledgement described in this section is different from the ACK defined in Section III. The acknowledgement of the delivery status of the control packets is sent back to the controller as mention above [13]. While in Section III, the control packets received at the plant is used as ACK at the sensor node to reduce the number of access times, and hence to improve the performance of the MAC protocols. In this paper, the communication protocols considered does not provide acknowledgement of successful delivery of the control packets to the controller. As in UDPlike protocols, estimation and control are coupling. In the following, a heuristic and computationally efficient scheme of estimation and control is considered.

Using UDP-like protocols and similarly derivation in [13], the optimal state estimator can be formulated as:

$$
\begin{aligned}
\boldsymbol{x}_{k+1 \mid k}= & \boldsymbol{A} \hat{\boldsymbol{x}}_{k \mid k}+\bar{\gamma} \boldsymbol{B} \boldsymbol{u}_{k}^{c}+(1-\bar{\gamma}) \boldsymbol{B} \boldsymbol{u}_{k-1}^{a}, \\
\boldsymbol{P}_{k+1 \mid k}= & \boldsymbol{A} \boldsymbol{P}_{k \mid k} \boldsymbol{A}^{T}+\boldsymbol{Q}+ \\
& (1-\bar{\gamma}) \bar{\gamma}\left[\boldsymbol{B}\left(\boldsymbol{u}_{k}^{c}-\boldsymbol{u}_{k-1}^{a}\right)\left(\boldsymbol{u}_{k}^{c}-\boldsymbol{u}_{k-1}^{a}\right)^{T} \boldsymbol{B}^{T}\right], \\
\boldsymbol{K}_{k+1}= & \boldsymbol{P}_{k+1 \mid k} \boldsymbol{C}^{T}\left(\boldsymbol{C} \boldsymbol{P}_{k+1 \mid k} \boldsymbol{C}^{T}+\boldsymbol{R}\right)^{-1}, \\
\hat{\boldsymbol{x}}_{k+1 \mid k+1}= & \hat{\boldsymbol{x}}_{k+1 \mid k}+\delta_{k+1} \boldsymbol{K}_{k+1}\left(\boldsymbol{y}_{k+1}-\boldsymbol{C} \hat{\boldsymbol{x}}_{k+1 \mid k}\right), \\
\boldsymbol{P}_{k+1 \mid k+1}= & \boldsymbol{P}_{k+1 \mid k}-\delta_{k} \boldsymbol{K}_{k+1} \boldsymbol{C} \boldsymbol{P}_{k+1 \mid k} .
\end{aligned}
$$

As shown in (8), the error covariance $\boldsymbol{P}_{k+1 \mid k}$ depends on the control input, implying that the separation principle does not hold. Here, an ad hoc procedure is deployed by 
(a)

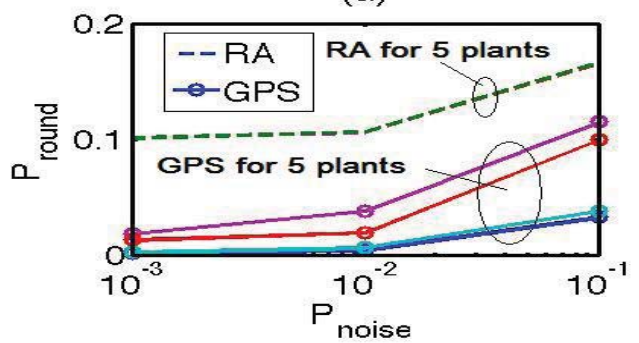

(c)

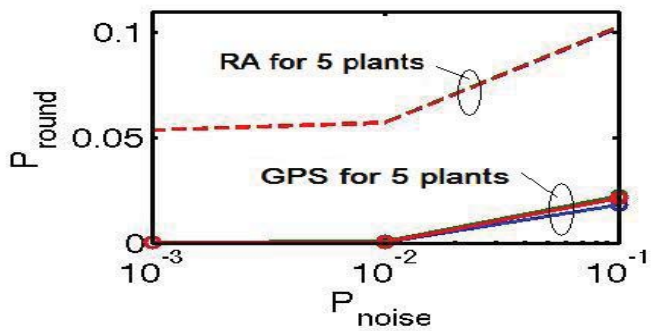

(b)

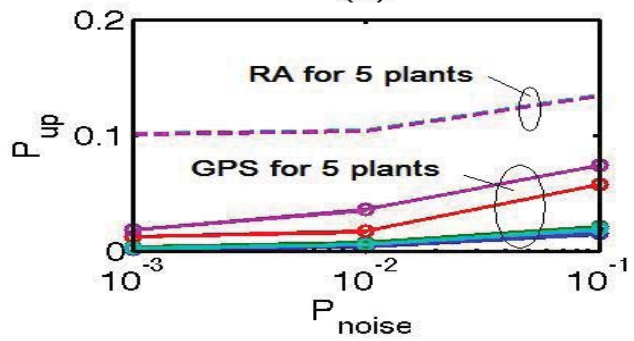

(d)

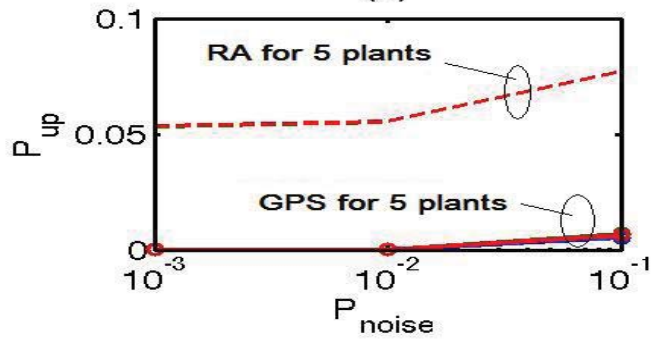

Fig. 6. Probability of packet loss as a function of $P_{\text {noise }}$ for GPS and $\pi$-persistent RA based MAC. (a) $P_{\text {round }}$ for round-trip, (b) $P_{u p}$ for uplink channel as $K=5, N=34$ and $N_{\text {fes }}=25$; (c) $P_{\text {round }}$ for round-trip, (d) $P_{u p}$ for uplink channel as $K=3, N=27$ and $N_{\text {fes }}=18$.

approximating the error covariance $\boldsymbol{P}_{k+1 \mid k}$ as:

$$
\boldsymbol{P}_{k+1 \mid k}=\boldsymbol{A P}_{k \mid k} \boldsymbol{A}^{T}+\boldsymbol{Q},
$$

where the last term in (8) is neglected assuming $0 \ll \bar{\gamma}<1$.

A linear control law is considered:

$$
\boldsymbol{u}_{k}^{c}=L \hat{\boldsymbol{x}}_{k \mid k},
$$

where $L$ is the linear quadratic regulator (LQR) feedback gain for the linear stochastic system (1)-(3) in the case of perfect data packet transmissions, i.e., $\delta_{k}=\gamma_{k}=1$ for $k=1,2, \ldots$. The state estimate $\hat{\boldsymbol{x}}_{k \mid k}$ can be estimated by the modification of the standard Kalman filtering in (7) and (9)-(12).

\section{NUMERICAL EXAMPLES}

Consider a NCS with multiple plants closed through the shared wireless network. By the use of (5), it gives $T_{a c k}=7 T_{t r}$ assuming $T_{p}=2 T_{t r}$ for $m=2$. The performance with respect to the probability of packet loss $P_{\text {round }}$ and $P_{u p}$ of the GPS and $\pi$-persistent RA based MAC protocols with ACK was examined. Furthermore, the control performance of the NCS was studied under the GPS and RA based MAC protocols with the proposed estimator/controller.

\section{A. Performance of MAC protocols}

Consider $K=5$ plants. For GPS, we have $p=q=5$ and $L=25$. For $\pi$-persistent RA, we have $\pi=1 / 5$. Fig. 6 (a) and (b) depict $P_{\text {round }}$ and $P_{u p}$ as a function of $P_{\text {noise }}$ for $N_{\text {fes }}=25$ ( $N_{t r}=13$ ), respectively. It can be seen that, the GPS outperforms the $\pi$-persistent RA. It is also noted that under the RA scheme, each plant has similar performance. While under the GPS scheme, plants using different sequences could have different performances as shown in Fig. 6 (a) and (b). The user using the first sequence, AS1, has the best performance as the first sequence has its superiority over other sequences. Therefore, in practice, we can assign the first sequence to the user with the highest priority so as to guarantee its performance. Consider $K=3$. For GPS, $p=q=3$ and $L=9$. For $\pi$-persistent RA, $\pi=1 / 3$. Fig. 6 (c) and (d) depicts the $P_{u p}$ and $P_{\text {round }}$ versus $P_{\text {noise }}$ for $N_{f e s}=18\left(N_{t r}=9\right)$. Similar performances of the two MAC schemes can be observed. Until now, we only illustrate the case when $m=2$. Given the same $N$, when $m=1$, i.e., $T_{p}=T_{t r}$, we have $N_{t r}=N_{\text {fes }}$ and there are more slots can be used to transmit. Hence the performance of both MAC protocols will be improved. However, the GPS still outperforms the RA.

\section{B. Control Performance of the networked controlled system}

To examine the performance of the NCS with the proposed MAC protocol and the control law, consider a NCS consisting of $K=5$ identical pendulum-cart systems. With a sampling time $T_{s}=50 \mathrm{~ms}$, the linearized model of a pendulum-cart embedded in the networked scheme is given as:

$$
\begin{aligned}
\boldsymbol{x}[k+1] & =\left[\begin{array}{l}
1.0402,0.0507,0,0 \\
1.6175,1.0402,0,0 \\
-0.0005,0,1,0.05 \\
-0.0206,-0.0005,0,1
\end{array}\right] \boldsymbol{x}[k]+\left[\begin{array}{c}
-0.0024 \\
-0.0970 \\
0.0008 \\
0.0306
\end{array}\right] \gamma_{k} u[k]+\boldsymbol{w}(k), \\
\boldsymbol{y}[k] & =\delta_{k} \boldsymbol{x}[k]+\boldsymbol{v}_{k},
\end{aligned}
$$

where $\boldsymbol{w}(k) / \boldsymbol{v}(k)$ and $\boldsymbol{x}_{0}$ are independent, zero mean Gaussian random vectors with covariance matrices $10^{-4} \boldsymbol{I}$ and $10^{-2} \boldsymbol{I}$, respectively. The state estimator in (7) and (9-12) is deployed 


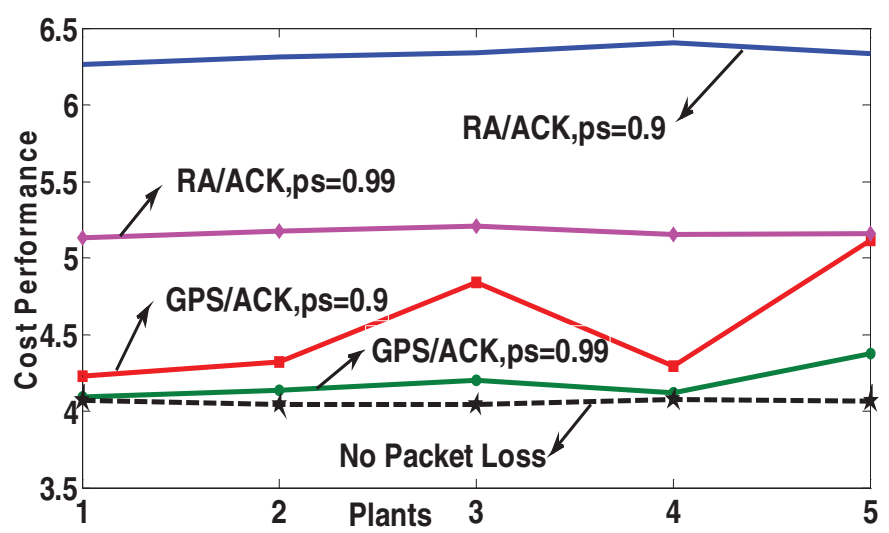

Fig. 7. Infinite horizon cost under GPS and RA based NCSs with $K=5, N_{\text {fes }}=25, P_{\text {noise }}=0.1,0.01$ and $M=4000$.

with $\boldsymbol{Q}=10^{-4} \boldsymbol{I}$ and $\boldsymbol{R}=10^{-4} \boldsymbol{I}$. The control policy in (13) is utilized with $\mathrm{Q}_{1}=\operatorname{diag}\{50,40,50,20\}$ and $Q_{2}=5$, where

$$
\mathbf{L}=[-40.7351,-7.5004,-2.1861,-3.5544]
$$

is the optimal LQR feedback gain for the linear stochastic system (14) with ideal packet transmission, i.e., $\delta_{k}=1$ and $\gamma_{k}=1$ for $k=1,2, \ldots$.

Fig. 7 depicts the infinite horizon cost performance $J$ for the GSP and $\pi$-persistent RA based NCS as $K=5$, where $M=4000$ samples are considered to calculate the stage cost. The expectation operator in (4) is averaged over 200 iterations. It can be seen that the control performance of the GPS based networked system is much better than that of the RA based system as $P_{\text {noise }}=0.1$ and $P_{\text {noise }}=0.01$. As shown in Fig. 8, similar control performance can be achieved when $K=3$ and $N_{\text {fes }}=18$. It is shown in Figs. (7) (8) that, as $P_{\text {noise }}=0.01$ with lower $P_{\text {round }}$ and $P_{u p}$, the cost performance of the GPS based NCS is close to that of the system with ideal packet transmission, especially when $K=3$.

\section{CONCLUSIONS}

In the paper, design of wireless MAC protocols is studied for time-critical networked control applications. The idea of protocol sequence was applied to arbitrated access to the shared wireless network. In particular, the GPS based MAC protocol with ACK was presented. It is shown that the GPS based protocol significantly outperforms the $\pi$-persistent RA MAC scheme with respect to the probability of packet loss. Using the control packets received at the plant as ACK, the performance of the GPS and RA based MAC protocols can be highly improved. A heuristic and computationally efficient estimation and control scheme was considered. Numerical results illustrate that the cost performance of the GPS based $\mathrm{NCS}$ is much better than that of the $\pi$-persistent RA based NCS. Efficient suboptimal estimation and control strategies will be further studied for the GPS based networked system. Design of efficient MAC protocols for NCSs will be studied, where multiple dynamical systems have various priorities.

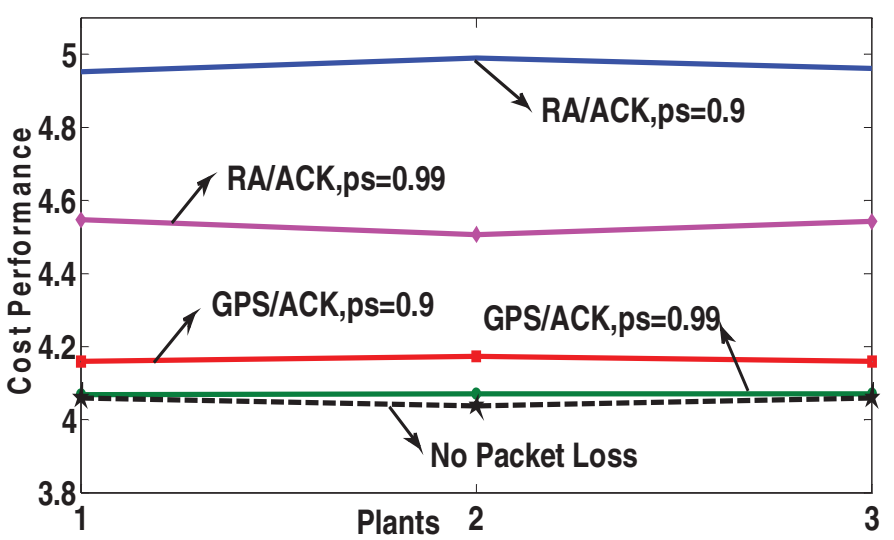

Fig. 8. Infinite horizon cost under GPS and RA based NCSs with $K=3, N_{\text {fes }}=18, P_{\text {noise }}=0.1,0.01$ and $M=4000$.

\section{REFERENCES}

[1] J. Baillieul and P. J. Antsaklis, "Control and communication challenges in networked real-time systems," Proc. of the IEEE, Vol. 95, No. 1, pp. 9-28, Jan. 2007.

[2] A. Suri, J, Baillieul and D. V. Raghunathan, Control using feedback over wireless Ethernet and Bluetooth, Handbook of networked and embedded control systems, pp. 677-698, Birkhauser Boston, USA, 2008.

[3] W. S. Wong and R. W. Brockett, "System with finite communication bandwidth constraints-II: stabilization with limited information feedback," IEEE Trans. Automat. Control, Vol. 44, No. 5, pp. 10491053, May 1999.

[4] L. Zhang and H.V. Dimitrios, "Communication and control co-design for networked control systems," Automatica, Vol. 42, pp. 953-958, 2006.

[5] X. H. Liu and A. Goldsmith, "Wireless medium access control in networked control systems," Proc. of American Control Conf. 2004, pp. 3605 - 3610, USA, 2004.

[6] C. Ramesh, H. Sandberg, and K. H. Johansson, "LQG and medium access control," Proc. of the 1st IFAC Workshop on Estimation and Control of Networked Systems 2009, pp. 328-333, Italy, 2009.

[7] C. Ramesh, Contention-based multiple access architectures for networked control systems, Thesis, KTH, Sweden 2011.

[8] W. S. Wong, "New protocol sequences for random access channels without feedback," IEEE Trans. Inform. Theory, Vol. 53, No. 6, pp. 2060-2071, June 2007.

[9] K. W. Shum, C. S. Chen, C. W. Sung, and W. S. Wong, "Shift-invariant protocol sequences for the collision channel without feedback," IEEE Trans. Inform. Theory, Vol. 55, No. 7, pp. 3312 3322, July 2009.

[10] K. W. Shum and W. S. Wong, "Construction and applications of CRT sequences," IEEE Trans. Inform. Theory, Vol. 56, No. 11, pp. 57805795, Nov. 2010.

[11] Y. Wu, K. W. Shum, W. S. Wong, Q. Su and L. F. Shen, "Safety messages broadcast in vehicular Ad Hoc networks based on protocol Sequences," submitted to IEEE Trans. Vehicular Tech., 2012.

[12] J. R. Moyne and D. M. Tilbury, "The emergence of industrial control netowrks for manufacturing control, diagnostics, ans safety data," Proc. of the IEEE, Vol. 95, No. 1, pp. 29-47, 2007.

[13] L. Schenato, B. Sinopoli, M. Franceschetti, K. Poolla and S. S. Sastry, "Foundations of control and estimation over lossy networks," Proc. of the IEEE, Vol. 95, No. 1, pp. 163-187, Jan. 2007.

[14] B. Sinopoli, L. Schenato, M. Franceschetti, K. Poolla and S. S. Sastry, "Optimal linear LQG control over lossy networks without packet acknowledgment," Proc. of the $45^{\text {th }}$ IEEE Conf. Decision \& Control 2006, pp. 392-397, USA, 2006.

[15] E. Garone, B. Sinopoli and A. Casavola, "On the effect of packet acknowledgement on the stability and performance of netowked control system," In Modelling, Estimation \& Control of Networked Complex Systems, pp. 1-16, 2009. 